accidental deaths (eg, violence, suicide and trauma). There is no apparent evidence of reduced mortality from CVD among lightto-moderate drinkers.

Conclusion Among Chinese adults aged 40-79 there was a J-shaped relationship between alcohol consumption and overall mortality.

\section{P2-335 CANCER MORTALITY AMONG CHRYSOTILE WORKERS}

doi:10.1136/jech.2011.142976k.67

${ }^{1} \mathrm{E}$ Yano, ${ }^{2} \mathrm{X}$ Wang, ${ }^{3} \mathrm{Z}-\mathrm{M}$ Wang, ${ }^{3} \mathrm{M}-\mathrm{Z}$ Wang, ${ }^{3} \mathrm{Y}$-J Lan. ${ }^{1}$ Teikyo University School of Public Health, Tokyo, Japan, ${ }^{2}$ School of Public Health and Primary Care, The Chinese University of Hong Kong, Hong Kong, China; ${ }^{3}$ West China Center of Medical Sciences, Sichuan University, Chengdu, China

Since the beginning of 1972, we have followed a group of workers in an asbestos plant in Chongqing, China. In the plant, chrysotile has been used to manufacture asbestos textile, asbestos cement, flooring materials, friction materials, etc. Previously, we performed a matched (1:5) case-control study by selecting cases of workers who developed lung cancer sometime between 1975 and 2001 and found the elevated risk of lung cancer associated with the exposure. In 2006, we summarised further follow-up results of the cohort and found that, in addition to the 132 deaths until the end of 25 th year, 101 workers were deceased during the last 10 years, and among them, 29 were from lung cancer (total: 51 lung cancers during the 35 years). Depending on the concentration of fibre of their workplace, we divided the workers into three levels of exposure. Compared to those in the administration and asbestos cement sections, workers in raw material and textile departments showed a higher HR for lung cancer $(3.9,95 \%$ CI 1.8 to 8.5$)$ in Cox proportional hazard model analysis. Similarly, HRs for total cancer and total death were 2.0 (1.2 to 3.3) and 1.5 (1.1 to 2.0), respectively. Multivariate analysis controlling for age, smoking, and working time did not change the results greatly. The results of the present study confirm the strong association of chrysotile exposure and lung cancer. Results of the fibre type analysis of asbestos would be presented.

\section{P2-336 FACTORS ASSOCIATED WITH CLASS III OBESITY AMONG ADULTS, NATIONAL TELEPHONE SURVEY (VIGITEL) BRAZIL, 2009}

doi:10.1136/jech.2011.142976k.68

\begin{abstract}
${ }^{1,2} \mathrm{R}$ Yokota, ${ }^{*}{ }^{1} \mathrm{~B}$ Iser, ${ }^{1} \mathrm{~L}$ Sardinha, ${ }^{1} \mathrm{~L}$ Moura, ${ }^{3} \mathrm{R}$ Claro, ${ }^{1} \mathrm{D}$ Malta. ${ }^{1}$ General Coordination of Non Communicable Diseases and Injuries, Ministry of Health, Brasilia, Distrito Federal, Brazil; ${ }^{2}$ Brazilian Field Epidemiology Training Program, Ministry of Health, Brasilia, Distrito Federal, Brazil; ${ }^{3}$ Center for Epidemiological Studies in Health and Nutrition, University of São Paulo, Sao Paulo, Sao Paulo, Brazil
\end{abstract}

Introduction In Brazil, class III obesity has increased from $0.18 \%$ (1975), to $0.33 \%$ (1989) and $0.64 \%$ (2003) in the last decades, according to National surveys. However, no information on recent trends in class III obesity prevalence is available in Brazil.

Objective To estimate the prevalence of class III obesity and its associations with sociodemographic and behavioural factors. Method: We analysed data from the Telephone-based Surveillance System of Risk and Protective Factors for Chronic Diseases (Vigitel) collected in 2009. Approximately 54000 adults were interviewed in all al state capitals and federal district. Individuals with body mass index higher than $40 \mathrm{~kg} / \mathrm{m}^{2}$ were identified as class III obese. Poisson regression models were used to evaluate the factors associated with class III obesity.

Result The prevalence of class III obesity was $1.1 \%$ ( $n=489 ; 95 \%$ CI 0.9 to $1.3 \%$ ) in 2009. Class III obesity was associated with sex $(\mathrm{PR}=2.36$; $95 \% \mathrm{CI} 1.4$ to 3.8$)$, dyslipidemia ( $\mathrm{PR}=1.9 ; 95 \% \mathrm{CI} 1.3$ to
2.8), hypertension ( $\mathrm{PR}=5.5 ; 95 \%$ CI 3.6 to 8.3 ), diabetes $(\mathrm{PR}=1.8$; $95 \%$ CI 1.2 to 2.7$)$, cardiovascular disease ( $\mathrm{PR}=2.2 ; 95 \% \mathrm{CI} 1.0$ to 4.6), watching television more than 3 hours/day ( $\mathrm{PR}=1.8 ; 95 \% \mathrm{CI}$ 1.1 to 3.0 ) and being current smoker ( $\mathrm{PR}=0.5$; $95 \%$ CI 0.3 to 0.8 ). When we included all associated factors in the model, sex $(\mathrm{PR}=2.0$; $95 \% \mathrm{CI}-1.2$ to -3.3 ; $\mathrm{p}$-value $=0.004)$, hypertension $(\mathrm{PR}=5.3 ; 95 \%$ CI 3.0 to 9.5 ; p-value $<0.001)$, watching television more than 3 hours/day ( $\mathrm{PR}=1.8 ; 95 \% \mathrm{CI} 1.1$ to 2.9 ; $\mathrm{p}$-value $=0.020$ ) and being current smoker $(\mathrm{PR}=0.5 ; 95 \%$ CI 0.2 to $0.8 ; \quad p$-value $=0.012)$ remained associated with class III obesity.

Conclusion Vigitel is an efficient tool to monitor class III obesity in Brazil. This study identified some factors associated with class III obesity that should be considered when developing prevention measures due to its relations with several chronic diseases.

\section{P2-337 SHORT TERM TRENDS OF OBESITY USING DATA OF THE TELEPHONE-BASED SURVEILLANCE SYSTEM OF RISK AND PROTECTIVE FACTORS FOR CHRONIC DISEASES (VIGITEL), BRAZIL, 2006 TO 2009}

doi:10.1136/jech.2011.142976k.69

${ }^{1,2} \mathrm{R}$ Yokota, ${ }^{*} \mathrm{~B}$ Iser, ${ }^{1} \mathrm{~L}$ Sardinha, ${ }^{1} \mathrm{~L}$ Moura, ${ }^{1,3} \mathrm{R}$ Claro, ${ }^{1} \mathrm{D}$ Malta. ${ }^{1}$ General Coordination of Non Communicable Diseases and Injuries, Ministry of Health, Brasilia, Distrito Federal, Brazil; ${ }^{2}$ Brazilian Field Epidemiology Training Program, Brasilia, Distrito Federal, Brazil; ${ }^{3}$ Center for Epidemiological Studies in Health and Nutrition, University of São Paulo, São Paulo, São Paulo, Brazil

Introduction Obesity has increased in high and low income countries, among all age groups. In Brazil, obesity has tripled in men and doubled in women from 1975 to 2003. Objective: To analyse short term trends in the prevalence of obesity among Brazilian adults from 2006 to 2009. Method: Data from Vigitel collected annually from 2006 to 2009 were analysed. Poisson regression models with obesity as the dependent variable and the year of the study as the explanatory variable were used to identify linear trends.

Result Obesity prevalence in Brazil increased from 11.4\% (95\% CI 10.8 to $12.0 \%$ ) in 2006 to $13.9 \%$ (95\% CI 13.1 to $14.7 \%$, p-value $<0.001)$ in 2009, without a significant difference between sex (2006 - female: $11.4 \%$, 95\% CI 10.6 to $12.2 \%$; male: $11.4 \%$; $95 \%$ CI 10.5 to $12.3 \%$; 2009 - female: $14.0 \%, 95 \%$ CI 13.1 to $14.0 \%$; male: $13.7 \%$; $95 \%$ CI 12.4 to $15.0 \%$ ). The higher prevalence of obesity was observed among adults with less educational level in all years studied (0-8 years of schooling: $2006-13.6 \%, 95 \%$ CI 12.5 to $14.6 \%$; $2009-16.1 \%$, $95 \%$ CI 14.6 to $17.6 \%$, p-value $=0.004 ; 9-11$ years of schooling: $2006-8.9 \%, 95 \%$ CI 8.2 to $9.6 \% ; 2009-11.6 \%, 95 \%$ CI 10.7 to $12.4 \%$, p value $<0.001$; $\geq 12$ years of schooling: $2006-9.6 \%$, $95 \%$ CI 8.6 to $10.6 \%$; 2009: 11.6 , $95 \%$ CI 10.6 to $12.6 \%$, $\mathrm{p}$ value $=0.007$ )

Conclusion The obesity increase among Brazilian adults demonstrates the need to improve prevention measures in Brazil. The agile and inexpensive data collection method used allowed it to have an important role in obesity plan control recently developed in Brazil.

\section{P2-338 ASSOCIATION OF BODY MASS INDEX WITH RISK OF AGE-RELATED CATARACTS IN A MIDDLE-AGED JAPANESE POPULATION}

doi:10.1136/jech.2011.142976k.70

${ }^{1} \mathrm{M}$ Yoshida, ${ }^{*} \mathrm{~K}$ Karita, ${ }^{2} \mathrm{M}$ Inoue, ${ }^{2} \mathrm{M}$ Imasaki, ${ }^{2} \mathrm{~S}$ Tsugane. ${ }^{1}$ Department of Public Health, Kyorin University School of Medicine, Mitaka-shi, Tokyo, Japan; ${ }^{2}$ Epidemiology and Preventive Division, Research Center for Cancer Prevention and Screening, National Cancer Center, Chuo-ku, Tokyo, Japan

Introduction Many epidemiological studies have demonstrated that body mass index (BMI) is associated with the risk of developing 
age-related cataracts. These reports have suggested that high and low BMIs can affect the onset or progression of age-related visual impairment. However, few prospective studies have examined this relationship in a general Asian population. Therefore, in this study, we investigated whether BMI was associated with increased risk of age-related cataracts by performing a 5 -year prospective populationbased study among a middle-aged Japanese population.

Methods This 5-year population-based study included 35365 men and 40825 women (aged 45-74), who were recruited onto the Japan Public Health Center-based Prospective Study (JPHC Study) and had not reported cataracts in baseline survey. The self-reported diagnosis of age-related cataracts was used in the analysis of this study.

Results At follow-up, 1004 men (2.84\%) and 1807 women (4.43\%) reported new diagnoses of age-related cataracts. The multivariate ORs for those in the lowest and the highest BMI category, compared with a BMI category of $21.0-22.9$ as a reference point (OR, 1.00), were 1.29 (95\% CI 0.93 to 1.79$)$ and 1.15 (95\% CI 0.96 to 1.39$)$ in men, and 1.23 (95\% CI 0.97 to 1.55$)$ and 1.19 (95\% CI 1.04 to 1.36$)$ in women.

Conclusion High and low BMIs have been suggested previously as the risk of age-related cataracts for Caucasian population in developed countries and the population living in developing countries respectively. However, the present large-cohort study showed that a $\mathrm{U}$-shaped association between BMI and incidence of cataracts in Japanese men and women.

\section{P2-339 ROLE OF NEUROMUSCULAR FUNCTION IN PREDICTING THE OCCURRENCE OF DISABILITY: THE ROAD STUDY}

\section{doi:10.1136/jech.2011.142976k.71}

${ }^{1} \mathrm{~N}$ Yoshimura, ${ }^{*}{ }^{1} \mathrm{~S}$ Muraki, ${ }^{1} \mathrm{H}$ Oka, ${ }^{2} \mathrm{H}$ Kawaguchi, ${ }^{2} \mathrm{~K}$ Nakamura, ${ }^{1} \mathrm{~T}$ Akune. ${ }^{1} 22 n d$ Century Medical and Research Center, The University of Tokyo, Tokyo, Japan; ${ }^{2}$ Department of Orthopaedic Surgery, The University of Tokyo, Tokyo, Japan

Introduction To assess the ability of neuromuscular functions to predict the occurrence of disability in Japanese general population. Methods The large-scale population-based cohort study entitled research on osteoarthritis/osteoporosis against disability (ROAD) has established three cohorts, from an urban, a mountainous, and a coastal area. The first follow-up survey was performed after 3 years and was attended by 2479 of 3040 baseline participants (81.5\%). From the entire ROAD data, we utilised the data of the elderly participants ( $\geq 65$ years) from the mountainous and coastal cohorts. Disability was defined by a relevant certification committee comprising clinical experts and physicians from each municipality. To evaluate the predictive ability of the indices of neuromuscular function, logistic regression analysis was conducted using occurrence of disability as an objective factor and the grip strength and walking speed for $6 \mathrm{~m}$ as explanatory factors after adjustment for age, gender, and body mass index.

Results Of the 914 participants who completed both baseline and first follow-up surveys, 88 individuals $(9.6 \%)$ were defined as new cases of disability over 3 years. Logistic regression analysis indicated that the higher the grip strength, the lower is the risk of disability (+1 kg; OR, 0.90; 95\% CI 0.86 to 0.95), whereas the lesser the walking speed, the greater is the risk $(+1 \mathrm{~s}$; OR, 1.15; CI 1.07 to 1.23).

Conclusions The cumulative incidence of disability among the elderly was $9.6 \%$ over 3 years. The indices of neuromuscular function may be potential predictive factors of the occurrence of disability over 3 years.

\section{P2-340 HARMONY IN FAMILY RELATIONS: EXPLORATORY ANALYSIS IN SEARCH OF A HARMONY SCALE}

doi:10.1136/jech.2011.142976k.72

Y-Y Yu, W Tam, T H Lam. ${ }^{*}$ The University of Hong Kong, Hong Kong, Hong Kong

Objective Relationship harmony is characteristic of Chinese values and has been increasingly identified as a factor that contributes to social stability. However, no existing survey instrument is available that assesses the concept of family harmony in a Chinese context. We aimed to develop an opinion scale that captures self-evaluated state of family harmony among Hong Kong Chinese.

Methods During June 2008-October 2008, we surveyed 587 Cantonese-speaking Hong Kong residents (aged 15 and above) randomly selected from a list of residential addresses. We collected demographic data and asked the participants how much they agree with a pool of 52 descriptive items on family relations, family functions, respect, loyalty, love and support, communications and conflict resolution. Most items were positively worded indicative good family relations except five reverse-coded items. Responses ranged from strongly agree (1) to strongly disagree (5). We used exploratory factor analysis with maximum likelihood extraction method.

Results Score distributions for all 52 items were similar, the majority of the participants "agreed" to most items. Mean score of the 52 items was $2.2(\mathrm{SE}=0.4)$. Exploratory factor analysis identified nine dimensions of the harmony construct with Eigen values above unity, which explained $59 \%$ of total variance, and two dimensions with Eigen values of at least two, which explained $48 \%$ of total variance.

Conclusion Small variation was found for each of the 52 opinion items on family relations harmony. After item reduction, more indepth interviews are needed to examine whether these opinion items truly reflect the state of family harmony.

\section{P2-341 THE PREVALENCE AND INFLUENCES ON AETIOLOGY OF ANGINA IN RURAL AND URBAN POPULATIONS IN A DEVELOPING COUNTRY: THE PERU MIGRANT STUDY}

doi:10.1136/jech.2011.142976k.73

${ }^{1} \mathrm{M}$ Justin Zaman, ${ }^{*}{ }^{2} \mathrm{C} \mathrm{L}$ de Mola, ${ }^{3,2} \mathrm{R}$ H Gilman, ${ }^{4} \mathrm{~L}$ Smeeth, ${ }^{2,4} \mathrm{~J} \mathrm{~J}$ Miranda. ${ }^{1}$ University College London, London, UK; ${ }^{2}$ CRONICAS Center of Excellence in Chronic Diseases, Universidad Peruana Cayetano Heredia, Lima, Peru; ${ }^{3} J o h n s$ Hopkins Bloomberg School of Public Health, Baltimore, USA; ${ }^{4}$ London School of Hygiene and Tropical Medicine, London, UK

Background Rural-to-urban migration in low- and middle-income countries causes an increase in individual cardiovascular risk. There are few data on prevalence of early stage coronary diseases such as angina in developing countries, while the understanding of the aetiology of angina is complicated by the difficulty in measuring it across differing populations.

Methods The PERU MIGRANT study was designed to investigate differences between rural-to-urban migrant and non-migrant groups in specific cardiovascular disease risk factors cross-sectionally. The Rose angina questionnaire was used to record chest pain, which was classified definite, possible and non-exertional. Mental health was measured using the General Health Questionnaire (GHO-12). Mantel-Haenszel ORs (adjusted for age, sex, cardiovascular disease risk factors and mental health) were used to assess the risk of chest pain in the migrant and urban groups compared to the rural group, and further to assess the relationship (age and sex-adjusted) between risk factors, mental health and chest pain. 